\title{
Nutrigenetics, Nutrigenomics and Precision Nutrition
}

\author{
Anna Meiliana ${ }^{1,2, *}$, Andi Wijaya ${ }^{1,2}$ \\ ${ }^{1}$ Postgraduate Program in Clinical Pharmacy, Padjadjaran University, Jl. Eijkman No.38, Bandung, Indonesia \\ ${ }^{2}$ Prodia Clinical Laboratory, Jl. Cisangkuy No.2, Bandung, Indonesia \\ *Corresponding author. E-mail: anna.meiliana@prodia.co.id
}

Received date: Jan 30, 2020; Revised date: May 29, 2020; Accepted date: May 29, 2020

\section{Abstract}

B ACKGROUND: Since our conception to death, we were permanently exposed to nutrition. Indeed, food intake is the key of the environmental factor that modulates our gene. Nutrigenomics focus on how common dietary chemicals altering an individual's genetic makeup including genome, proteome, and metabolome. While nutrigenetics refer to how the genetic variation gives different responses to nutrients.

CONTENT: Nutrigenomics applied the high-throughput genomic-related tools to find out the influence of nutrients on the genes' expression. While nutrigenetics (nutritional genetics) focus on the heterogenous response of gene variants to nutrients and dietary factors. Application

\section{Introduction}

Nutrition has a major impact on public health, not only for diseases prevention but also act to promote wellness and optimal aging through selected dietary recommendation. Nutritional environment and cellular/genetic are coinfluencing one toward another. Indeed, food intake is the key of the environmental factor that modulates our gene. Undeniable, we are all exposed permanently to food intake from our conception to death. In the 20th century, Garrod in his famous Croonian lectures about inborn errors of metabolism led us to the concept on phenotype determination as the result of nutrition and genetics interactions. After a century the idea keep ringing, involve current technologies of human genome sequencing, and nutrigenomics and nutrigenetics, integrated with system biology result in a precision nutrition as a relevant precise personal dietary change recommendation, thus will increase the motivation and sustain to whom the intervention is being delivered.

SUMMARY: Individual diet recommendation is not simple. Many factors and tools should be involved adequately. The application of integrated -omics approaches, together with nutrigenomic and nutrigenetics for novel biomarker discovering and precision nutrition tailoring were expected to develop a healthier lifestyle and behavior.

KEYWORDS: nutrigenetics, nutrigenomics, precision nutrition, metabolomics, system biology

Indones Biomed J. 2020; 12(3): 189-200 bring up the concept of nutritional genomics. Genetics is the study of heredity, the mechanisms of how the parents passes specific traits into the offspring, and how the variations in genes or genes combination explain the traits differences.(1) On the other side, genomics explores the genes response under different conditions including the environmental factors utilizing various technologies to examine large numbers of nucleotide sequences, genes, and proteins.(2) Nutritional genomics or "nutrigenomics" is a term that focus on how common dietary chemicals (i.e., nutrition including macronutrients, micronutrients and antinutrients) affect the balance of our health and disease by altering the expression and/or structure of an individual's genetic makeup including genome, proteome (the sum total of all proteins), as well as metabolome (the sum of all metabolites).(3,4) On the contrary, nutritional genetics 
or "nutrigenetics" refer to how the genetic variation gives different responses to nutrients. In current postgenomic era, coupling the genomics, transcriptomics, proteomics, metabolomics with bioinformatics and chemometrics, comprehensively manage and interpret as a complex data set commonly known as "functional genomics" or "system biology".(5) System biology developed the traditional approach of nutrition-metabolism model into a modern holistic view on gene-nutrient interaction, in which a significant fraction of all regulated genes and metabolites can be quantified concurrently.(6) The identification and characterization of gene variants was expected to generate a specific diet recommendation for individual regarding his responses to dietary components, termed as 'personalized nutrition' (PN).(7-10) If previously we were familiar with "personalized medicine", now it is extended to the field of nutrition. In the end, the molecular processes alteration will alter disease initiation, development, or progression.(3)

As a young science, nutrigenomics attracted a lot of attention and developed rapidly and widely. Earlier hypes tried to apply the science but raise unhelpful solutions. However, recent studies put a major advance in quantifying the contribution of genetic variation to a wide range of phenotypes, to clarify the nutrition-related phenotypes, especially for common complex diseases like obesity which cannot be explained just by single-nucleotide polymorphism (SNP) alone. Other factors may also be contributed as suggested by metagenomic studies about the gut microbiota unexpected, substantial effects which may be important on multiple aspects of human health, and for sure nutrition, again be involved as the most important mediator of the composition and function of our commensal flora. New tools, including stem cell-based approaches and genome editing, together with nutrigenomics and system biology have huge potential to transform the future of mechanistic nutrition research.(11) This approach then hold a big potential to change human dietary habits in order to achieve effective disease prevention and therapy.

\section{Nutrition in The Postgenomic Era}

Since the ancient time we believe that our diet will affect our health. For example, Phenylketonuria (PKU) is caused by a single gene mutation. Then, the affected individuals have to avoid amino acid phenylalanine diet. Another common example is people with lactose intolerance, means they cannot digest milk or milk products due to inactive lactase, the enzyme that breaks down lactose. In these people, the gene encoding lactase is "turned off" after weaning.(12) The science of nutrigenomic jumped-started since The Human Genome Project in 1990s sequenced the entire DNA in the human genome. By 2007 scientists found out that our food communicates to our genes, and this miscommunication will lead to diseases. What we eat delivered messages to our genes, drive a control on our metabolism, including tell our body to burn calories or store them. This control the messages and instructions delivered to our body and metabolism. Therefore, the interaction can be altered between the food and our body, lose weight, and optimize your health, as well as to modify the genes that affect the risk of getting illnesses like heart disease, cancer, osteoporosis and diabetes by food regimen. $(1,13)$

It will be ideal if we can take benefit from nutrition to optimize our cellular, tissue, organ and whole-body homeostasis. This shifting the nutrition research from Epidemiology and Physiology to Molecular Biology and Genetics then nutrigenomics was born. Nutrigenomics extracting the understanding of how nutrients or food bioactive at the molecular level, characterize gene products, their physiological function and their interactions at gene, protein, and metabolite level (Figure 1), as well as how it influences the gene expression (transcriptomics, proteomics and metabolomics), result in a nutrient-regimes on human health. The three of transcriptomics, proteomics and metabolomics can be combined into a Nutritional Systems Biology approach.(7)

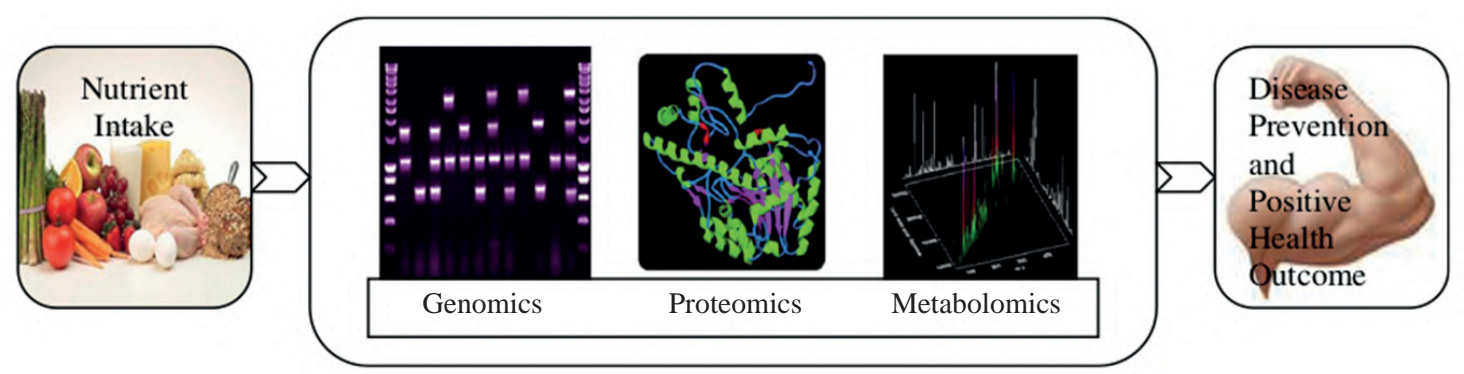

Figure 1. Nutrigenomics approach in disease prevention.(12) (Adapted with permission from Springer). 
Nutrigenomics backgrounded by two sides of paradigms, which could labeled as an applied science in the sight of nutritional pharmacology, in the context of genetic polymorphism and clinical experience, by using the microarray technology and integrated on an informatics platform in the sight of molecular biology. $(5,9)$ A healthy phenotype progress into a chronic disease via changes in gene expression, cause alterations in protein and enzymes activities. Ingested dietary chemicals participate either directly or not, in regulating gene expression. We can conclude that diet must be take part in shifting a healthy phenotype into sick since the initiation, progression until severity.(14,15) The most common chronic disease involving genotype-diet interaction is type 2 diabetes (T2DM), generally known occurs in sedentary, obese individuals and certain minority groups.(16,17) T2DM subjects advised to control their symptoms by increasing physical activities and reducing caloric intake especially fat (18) and also received such an environmental interventions and drug treatments. In the molecular level, nutrients can be considered as "signaling molecules" to change the genes, protein and metabolite expression via the appropriate cellular sensing mechanisms.(19) Afterwards, the question is what happened, when we do not eat wisely? On the genomic level, nutrients act as "signatures" that linked to the phenotype, especially in metabolic stress, which is the early phases of organ-specific insulin resistance occur.(20)

Chronic exposure of certain food components suggested to mediate the occurrence of chronic diseases. However, everybody tends to respond differently to diet. Nutrigenetics explain that genetic polymorphisms in apolipoprotein E, fatty acid desaturase, lipoxygenase-5, peroxisome proliferator-activated receptors (PPARs), apolipoprotein A1, apolipoprotein A2, apolipoprotein $\mathrm{A} 5$, and methylenetetrahydrofolate reductase have been associated with cardiovascular disease.(21) This means any gene-diet interaction occurred will affect to the diseases, and any gene variation either due to ethnicity, environment, disease/condition, genes, polymorphisms, nutrients will play a role.(20) The excitements about how diet modification will reduce the risk of diet-related diseases bring the science of bioinformatics, nutrition, molecular biology, genomics, epidemiology, and molecular medicine to unfold the role of nutrition on gene expression. Figure 2 is highlighting the known and unknown information in the field of nutrigenomics.

\section{Systems Biology in Nutrigenomics and Nutrigenetics}

Biological networks including the interactions between genes, proteins and metabolites which are coordinated to regulate cellular processes. The cells-to-cells interaction yield in tissue response, and different cell types interaction to synchronize, result in physiological response in organs. (22) System biology can be simply defined as the study of biological systems. System biology aim to understand the complexities of multidiscipline involved such as mathematics, engineering, computer science, physics, chemistry and biology in a comprehensive process.(23) Composed of molecular components, system biology combines the data from experimental basis and mathematical model to understand the network behavior between gene
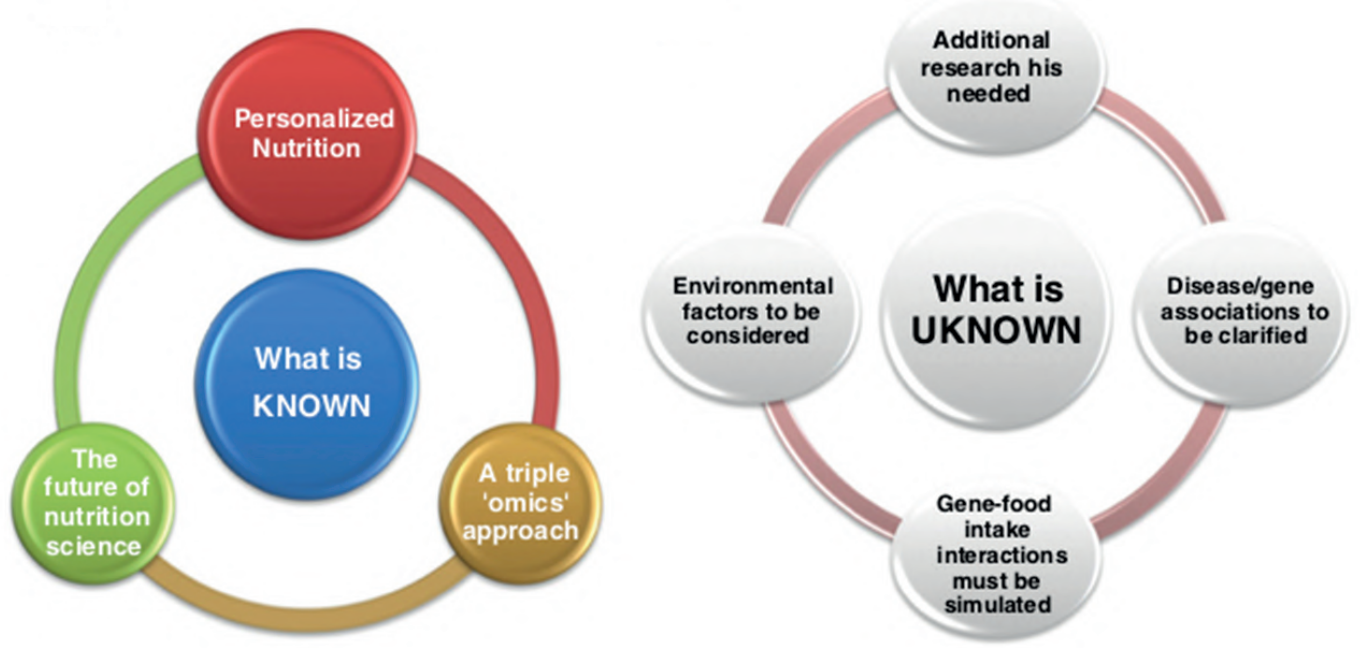

Figure 2. A schematic depiction of what is "known" and what is "unknown" in the field of nutrigenomics, highlighting the findings and challenges that emerge for the field of nutrigenomics.(20) (Adapted with permission from Elesvier). 
protein and informational pathways. $(23,24)$ Merging system biology with computational techniques can be employed to study the networks that mediate physiological system dynamics in cellular or organ level, and in the end, we can understand how nature engineered our biological systems through evolution, measuring all differentially perturbed states, and wrap up the basic principle that govern them (Figure 3).(25,26) Then it is possible for us to create a simulation of the physiology of the cell. $(27,28)$

Our metabolism could be expressed as a transient steady state in the cellular biosynthesis dynamics. Proteins function either as enzymes, receptors, transporters, channels, hormones and other signaling molecules. It provides structural elements for cells, organs or the skeleton. On the contrary, metabolites serve a broad role in cell functions. It usually rapidly "converted" in enzymatic and chemical reactions, to be the building blocks for macromolecules, or role as transient energy-storage. Hence, metabolites reaction, identification, and quantification are important in system biology, and metabolomics become an important study to understand the entire set of metabolites in a cell, tissue or organ sample.(29-32)
The advancement of -omics technology shifting from the classical analytical into system biology. In this way, any human biological process in interest, especially system, could be studied as comprehensive networks of functionally interacting macromolecules and reactions. By functional genomics we can identify the genes and their products in particular modules extensively, including to find the relationship between them.(33) The goals of systems biology are: interpret the component of one biological system and their structures. This can be genes, proteins, or metabolites; explain the dynamic of the system, including both quantitative and qualitative analysis and also the theory/model construction based on powerful prediction capability; find out the control methods of the system; and understanding of design methods of the system. Figure 4 shows an example of systems biology iterative research.

When we eat, a complex mixture of various bioactive chemical compounds in different compositions was taken, chronically this will send a multitude of biological effects. These responses are mediated through the effector genes, and induce interaction between genes. The concentration and activity of enzyme, and metabolic concentration

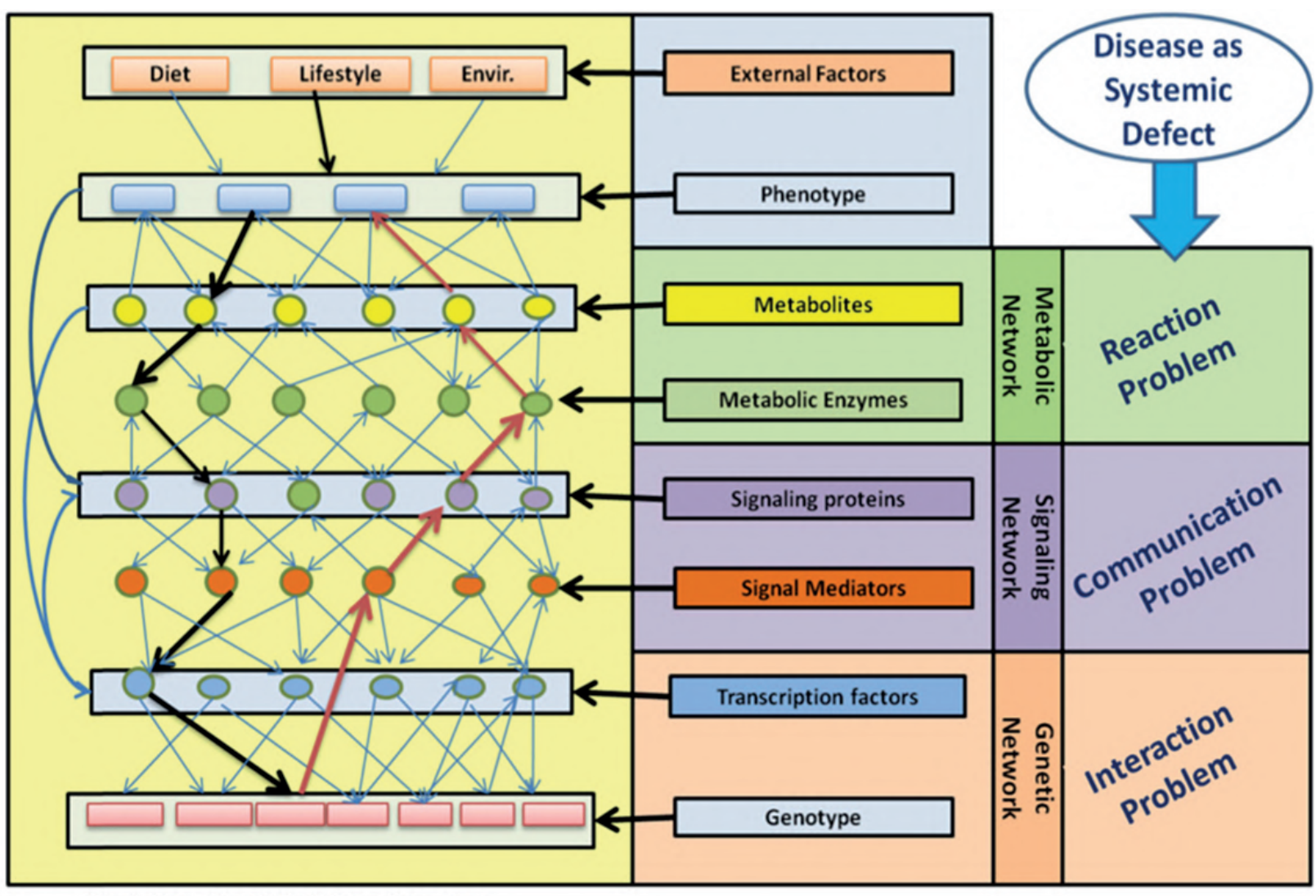

Black Lines- Signal, Red Lines- Response

Figure 3. Schematic diagram showing the typical coordination of genetic, signaling and metabolic networks for generating a phenotypic response.(22) (Adapted with permission from Springer). 

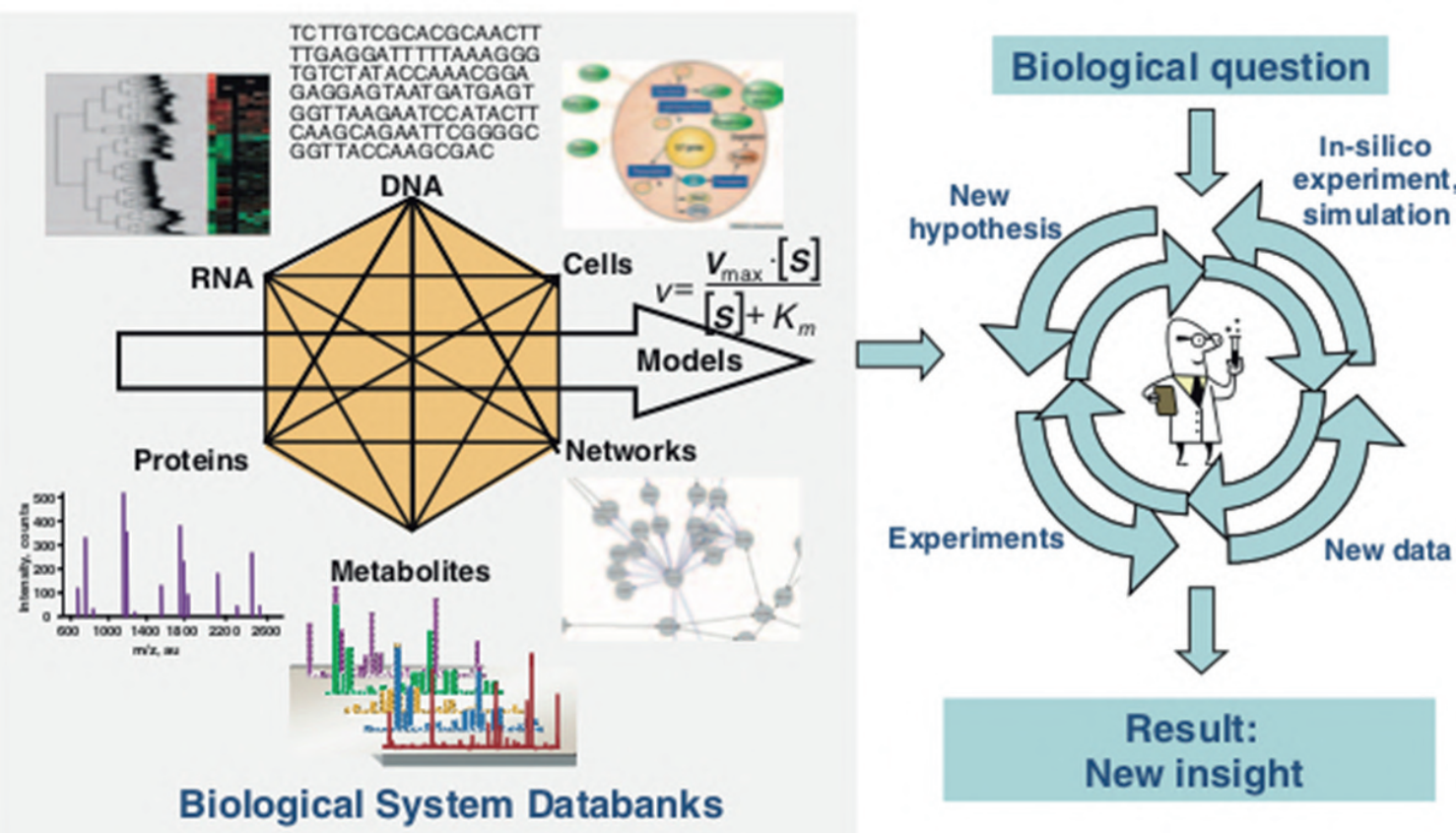

Figure 4. Systems biology iterative research.(33) (Adapted with permission from Elsevier).

changes (Figure 5). Besides of the low classical detection limit, multiple minor changes taken together, processed by new bioinformatics approaches result in a new sense of sensitivity in -omics technology. Multivariate statistical methods play a good role to analyze those "holistic" data sets to carefully describe what is healthy and how is the early changes.(7) Thus, nutritional science exploration no longer depend on the disease end point but work more with normal physiologic conditions and its dynamic and reversible situations to determine the disease prevention.

Nowadays, food and nutrition gain their recognition in human health and especially for illness prevention. Modern lifestyle's diseases such as diabetes, heart disease and cancer were proven to benefit from change of dietary patterns. Genetic polymorphisms mostly affect the risk of disease, somehow dietary intake and nutritional status involved. Nutritional researches can utilize the integrative biological study from the molecular level to whole body studies with systems biology approach to reach a more comprehensive understanding about the link between diet and health. In particular, applied system biology in nutrigenomics and nutrigenetics as a new discipline investigates how nutrients interact with humans, counting the role of genetic factors and mediate new insights into human health, formulating natural ways of disease prevention thus finally have significant positive impact on our quality of life.(33)

\section{The Concept of Precision Nutrition}

Like drugs, nutrient interact and modulate molecular mechanisms underlying an organism's physiological functions. Due to different genetic variants, individual respond to lifestyle intervention must be different too in how dietary components are absorbed, metabolized and utilized.(34,35) Thus, specific dietary advice based on genotype data should be more compelling than the general recommendations.(36) Personal recommendation on diet modifications will be arranged based on the genotype to optimize wellness, known as PN. Nutritional care should improve their standards based on the recognition of diverse individual nutritional needs and responses, in term personalization.(37)

Nutritional sciences conventionally observed how nutrients affects our body in "average" response, without considering that each individual can respond differently. The advance in nutrigenetics and nutrigenomics unravel the complex relationship between nutritional molecules, genetic variants and biological system create a personalized regiment for individuals. PN can be defined as a concept to adapt specific nutrition regime based on each individual need. Based on the combination of individual's fixed traits including genetic, environmental and lifestyle factors, PN develop an effective approach. Single approach by 


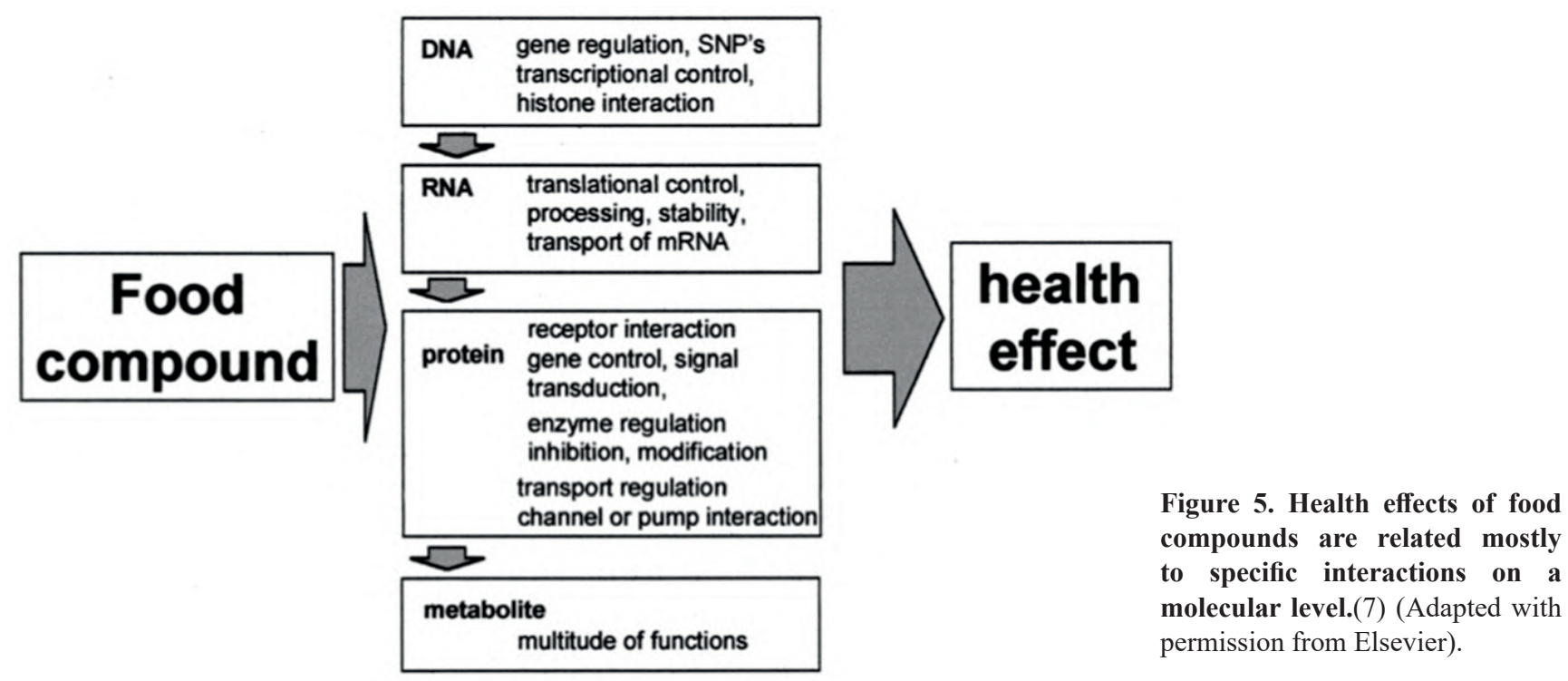

genetics turns out not very effective without exploring the environmental and/ or behavioral 'lifestyle' variance within each person, in other word that what we do and where we are may be more important than what we are. PN should also be adjusted dynamically, by considering the varying physiological demands and requirements over time.(38) Regarding those, precision nutrition become more appealing and prospect rather than $\mathrm{PN}$.

Since the publishing of OMICS, a journal of integrative biology published its special issues in 2008 and 2009 about nutrigenomics (39), the field have been so much evolved, as genomics-guided and individuallytailored diets have also proliferated, by considering the benefits on long-term health outcomes and/or personal lifestyles.(40,41) Gene transcription and the protein structure were not always influenced by SNPs. Some nitrogenous bases doesn't lead to change in amino acids to lead protein expression.(42-44)

$\mathrm{PN}$ is an important part of personalized medicine and healthcare improvement by establishing nutritional recommendations guidelines for specific subgroups based on individual genetic profile, phenotype, health status, food preferences and environmental characteristics. $(44,45)$ Started from individual health promotion and maintenance, -omics technology will expand into effective public health strategies on diet therapy. $(46,47)$. So, omics tools have to immediately develop into improved nutrigenetics, nutrigenomics and nutria-epigenetics.(48) Relevant to nutrigenomics and nutrigenetics, nutria-epigenetics investigates the epigenetic, or the chromatin structure and DNA modifications that do not alter the underlying DNA sequence, but affect gene expression.(49)
Interactions of metabolic, environmental, social and genetic factors together with biological and cultural variations, including food intolerances, preferences and allergies can determine individual response to nutrient. Individual's genome analysis can distinguish responders from non-responders to dietary interventions and treatments.(50) By these knowledge and integration, International Society of Nutrigenetics/Nutrigenomics (ISNN) recommended that PN could be applied at three levels: general conventional nutrition guidelines, based on population's age, gender and social determinants; individualized nutrition in the term of individual phenotypic current nutritional status (e.g., anthropometry, biochemical and metabolic analysis, physical activity, among others); and genotype-based nutrition direction, including rare or common gene variation. (51) Such information ideally will ensure that health-care professionals, including dietitians, physicians, pharmacists and genetic counselors, have sufficient knowledges about nutrigenetics and nutrigenomics and could decide the most appropriate precision nutrition which integrates phenotypical and genotypical issues, social, environmental and metabolic factors.(52-54)

\section{Nutrigenomic, Nutrigenetic, and Precision Nutrition}

A proper diet is clearly giving benefit not only for avoiding deficiency diseases but also to optimize the wellness and aging prevention. Therefore, every individual has different risks and benefits, and needs different diet regimen. We have a better understanding about molecular basis of how 
food activities and individual responses interact recently. Nutrigenomics is the core in this new knowledge to be applied in daily life.(55) The term "nutrigenomics" here encompasses both nutrigenetics and nutrigenomics. Nutrigenomics aimed to bridging genome research, biotechnology and molecular nutrition research and providing an advanced development in the field of nutrition for health, giving a comprehensive information of food influences in our homeostatic system, estimating the beneficial and adverse potential effects of foods in precocious phases, even before the initiation of a disease.

Chronic diseases such as cardiovascular disease (CVD), cancers, diabetes, neurological disorders, obesity, osteoporosis, and a variety of inflammatory disorders generally take benefit from nutrigenomics application. These diseases represent homeostasis disturbances, and environmental signals such as bioactive dietary components are the most potent homeostasis-influencing agents, include both nutrient and non-nutrient factors. Cellular sensor system detects these environmental signals and carry on activating genes and protein expression and, thereby, metabolite expression, then of course affect the physiological function. A comprehensive research of nutrigenomics will achieve a complete molecular data on these interactions and how they influence on homeostasis, the communication mechanism of the genes involved in signal transduction processes and how they role in related proteins and metabolites. This information could be extended to explore new biomarkers that serve as early warning sign of homeostasis disruption, and to find a pivotal point in diseases progression. Different from the single-gene disorders, chronic diseases usually are the results of multiple genes and multiple genes variants interacting with multiple environmental factors, including the complex interaction of diet and genes. Sure this will be a great challenges, but hold a greater promises to improve global health.(2) Researchers wishes to completing this nutrigenomic data translation into accurate prediction related to adversary health effects of dietary components, and reach the goal of prevention of diet-related diseases. In a nutrigenomics perspective, nutrients regard as dietary signals to activate cellular sensor system to influence genes, protein, and finally metabolite production. This course remarks a "dietary signatures", which is specific on different cells, tissues, and organisms, and could explain how nutrition influences homeostasis.(8)

Some strategies were developed to utilize the dietary signatures. First is the traditional hypothesisdriven approach, which is using genomics tools, such as transcriptomics, proteomics and metabolomics to identify specific genes and proteins expression by nutrient influences, and which regulatory pathways the diets affect homeostasis. The second strategy is not very practical at current stage, is the system biology approach: by listing the gene, protein and metabolite signatures related with specific nutrients in a catalogue, thus could provide 'early warning' for nutrientinduced changes to homeostasis. Combining these two strategies, we will obtain the detailed interaction between nutrition and the genome molecular data and strategy for human nutrition.(8) Therefore, the goals of nutrigenomics research can be defined as: identify the transcription factors that function as nutrient sensors and their target genes; elucidate the signaling pathways involved, and characterize the main dietary signals.

Precision Nutrition 4.0 involves Big Data management and ethic foresight analysis for the confluence of agrigenomics, nutrigenomics, nutriproteomics and nutrimetabolomics. While precision nutrition goes further by completing the genomic information with phenotypical, cultural, behavioral and lifestyle preferences, yielding in universal guide and personal advice for health maintenance and disease management.(56) However, no matter how ideal the dietary treatment is, the greatest challenge here is that of motivating individuals to change their dietary habits and behaviors. Individual's motivation still holds the main determination for the success of personalized diets.(48) Eating involves psychosocial factors, so individualized diet regimen based only on genetic information could be stressful. However, compared to traditional nutritional advice, nutrigenomic advice is better understood and more likely to be followed $(57,58)$, and also proven to be beneficial in long-term weight control (59) as the review within the Food4me project (60).

Individual preferences on food choices is complex, including sensory likes, availability and cultural habits.(61) New biomarkers to translate such issues will support PN to recognize consumer demands in the nutritional market.(62) It is necessary to collaborate the food industry with culinary practice to create a preferable personalized nutrition. $(10,62,63)$ Individualized nutritional recommendations should be makes sense and not complicated, such as the advice to eat five servings of fruits or vegetables per day. (64) Once gene-nutrient interaction is involved, nutritional advice cannot anymore be 'one size fits all' such as longchain omega-3 polyunsaturated fatty acids (PUFAs). In facts, individuals with the A allele for the Apolipoprotein A1 (APOA1) polymorphism ( $\mathrm{G}>\mathrm{A})$ get benefit after increased consumption of PUFAs and show an increase in highdensity lipoprotein (HDL) cholesterol levels, while people 
with GG genotype have the opposite.(6) Another example, a high-salt diet only induce higher systolic blood pressure to those with major risk alleles for serum- and glucocorticoid inducible kinase 1 (SGK1).(65)

Advances in laboratory techniques in PNincluding mass spectrometry, chromatography, electrophoresis, microarrays and magnetic resonance spectroscopy (66) support scientist with a holistic approach to improve nutritional interventions based on both nutrition and metabolism consideration and enabling customization of precise diet according to individual needs (67), as also identify individuals who will benefit from precise intervention strategies (68). Omics technologies make it possible to stratify the disease risks, encompassing proteomics, lipidomics and metabolomics for biomarker assays, and analyses of food bio-actives and compounds, to develop a customized diet. It was also need to establish guidelines for genetic tests and analysis regulation, to protect individuals from harmful services, involving analytical regulations (validating if the genetic tests are accurate to identify genes and polymorphisms), clinical validity (assess the accuracy of interpretation and association with clinical status) and clinical utility (evaluate the possibility of reaching a desired clinical result with a recommended intervention) as factors of consideration. $(69,70)$

\section{Precision Nutrition in The Prevention and Management Of Diseases}

Basic nutritional science has been grown and the findings translated extensively into meaningful and clinically relevant dietary advices. Many factors need to be considerate in designing personalized and unbiased nutritional solutions for individuals become current main challenges of clinical nutrition, especially to implement these new findings at the population level.(71)Chronic and metabolic diseases become the main goal for tailored nutritional recommendations (38). Ideally, PN recommendation should be comprehensive and dynamic fitly flowing with individual's internal and external environment throughout life. Thus, PN need more than just genetic factors but also dietary habits, food behavior, physical activity, the microbiota and the metabolome as described in Figure 6. Nutrigenomic research in this aim focus on the role of metabolic stress in generating metabolic syndrome, the collection of phenotypes combining inflammation, metabolic stress, insulin resistance, and diabetes (19), with ambition to supplement pharmacological therapy, and restoring the metabolic impairment. The earlier

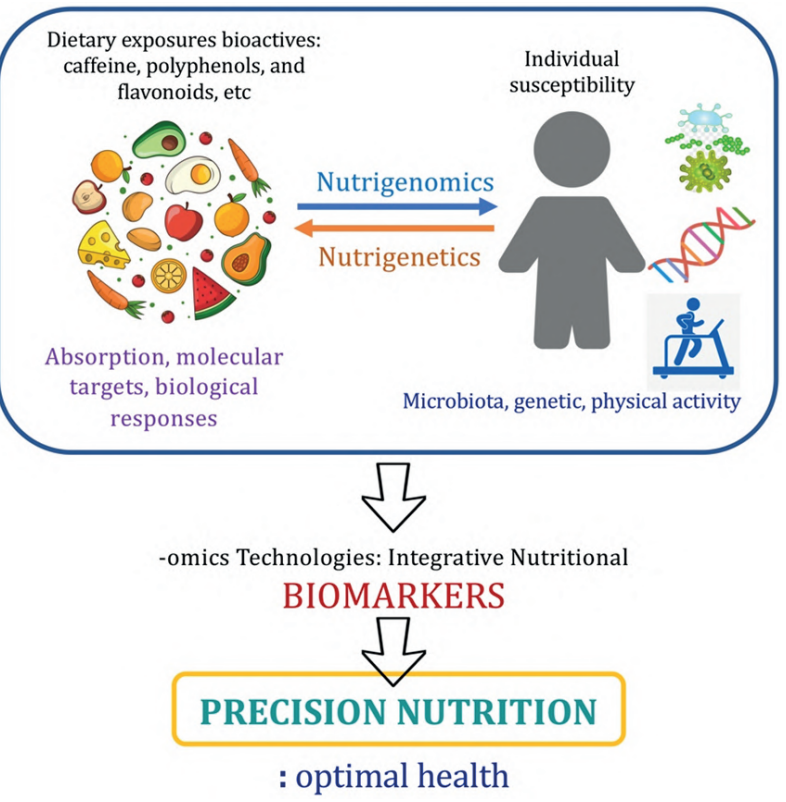

Figure 6. Steps involved in tailoring a precision nutrition to reach optimal health.

we could detect the onset of disease, or even the pre-disease state of the metabolic syndrome, or a condition known as metabolic stress, the higher possibility nutrition could take role in fixing this. For this achievement, new genomicsbased phenotypical biomarkers will be helpful.(72)

Nutrients influence on gene expression happens through a main agent called transcription factors which act as nutrient sensors in metabolically active organs, such as the liver, intestine, and adipose tissue. It changes the level of DNA transcription of specific genes in response to nutrient changes. The most important group of the nutrient sensors is nuclear receptor superfamily, which is ligand-activated transcription factors that have 48 members in the human genome, and play diverse roles in cell differentiation/ development, proliferation, and metabolism, including the pathogenesis of diseases. The receptors in this superfamily bind nutrients and their metabolites. For example, nuclear receptors, such as peroxisome proliferator activator receptor (PPAR)- $\alpha$ (binding fatty acids) or liver X receptor $\alpha$ (binding cholesterol metabolites), bind together with retinoid $\mathrm{X}$ receptor as heterodimers to specific nucleotide sequences (response elements) in the promoter regions of a large number of genes. Upon binding their respective ligand, nuclear receptors undergo a conformational change, and the corepressors dissociated, recruit coactivator proteins to enable transcriptional activation.(72)

Chronic diseases such as cardiovascular disease, metabolic syndrome, and cancer are partly mediated by chronic exposure to certain food components, so changing 
food habits considered to be useful as the prevention strategy. Studies to find the "signatures" of health and diseases, the causal relationship how those bioactive dietary components could role in diseases prevention can only be assessed by long-term intervention trials, with large study populations of patients and controls which are time-consuming and costly. However, it will give a great benefit for a more complete phenotyping of humans and the ability to monitor health status using a noninvasive tool utilizing omics technology. Metabolomics is the study of total endogenous and exogenous metabolites in a cell, organ, or body fluids. (3,7,73-76) Through metabolomics, individual metabolite profiles could be mapped, such as complete plasma lipid (i.e., cholesterol, triglycerides) and vitamin profiles.

The sensitive and well validated tool in nutrigenomics is transcriptomics. It employs microarray analysis to count the sum of messenger RNA's transcripts. By this technique, the expression levels of thousands of genes are enabled to determine at once, within one study. Many chronic diseases are multifactorial disorders caused by multiple genetic and environmental factors. Multiple susceptibility genes involved in multigenic or polygenic diseases. A similar disease phenotype is possible due to different combinations of gene variants, and a genetic variation can induce several dietary components to modulate the phenotype, make it more complicated for the analysis.(77)

Regarding obesity and metabolic syndrome, recent studies revealed the important impact of macronutrient intake correlated to genetic markers in metabolic health, fat mass accumulation or body composition. The environmentgene correlation opened the door to design efficiently diets based on the individual genetic makeup. Recent work imply a genetic risk score (GRS) to predict obesity and the impact of macronutrient intake.(78) GRS was counted as a sum of a set of 16 genetic variants (according to the number of risk alleles for each variant) known associated with obesity obesity (rs9939609, FTO; rs17782313, MC4R; rs1801282, PPAR- $\gamma$; rs1801133, MTHFR and rs894160, PLIN1), and lipid metabolism (rs1260326, GCKR; rs662799, APOA5; rs4939833, LIPG; rs18005881, LIPC, rs328, LPL; rs12740374, CELSR2; rs429358 and rs7412, APOE; rs1799983, NOS3; rs1800777, CETP and rs1800206, PPARA). The GRS validated. i.e., high risk group (subjects having more than 7 risk alleles) showing increased body mass index (BMI) $\left(0.93 \mathrm{~kg} / \mathrm{m}^{2}\right.$ greater BMI), body fat mass (BFM) $(1.69 \%$ greater BFM), waist circumference (WC) (1.94 cm larger WC) and waist-to-hip ratio (WHR) (0.01 greater WHR). They found significant interactions between macronutrient intake and GRS prediction values.(78)
Another clinical study involving gene-macronutrients interactions to develop GRS using BMI-associated SNPs as basis found the risk of obesity increased by high intake of sugar-sweetened beverages (78-81), fried foods (82) or saturated fatty-acids (83). All these studies suggest that someone's body weight was influenced by the common polymorphisms at certain loci, combined with the diet exposure. The scientific community recognizes that in the future, nutrigenomic solely won't be enough for PN.(44) Personalized diet tailoring need more than just nutrigenetics, but also lifestyle including physical activity habits, metabolomics or gut microbiomics which contribute significantly.(84-86)

Chrono-nutrition is the study of the interaction of food component with circadian clocks, thus meal time will affect metabolic process. $(87,88)$ This variant too, should be included in tailoring the personalized diet. Therefore, scheduled and personalized nutrition programs also aimed to improve the metabolic disorders in obesity. Cardiometabolic disease, as one of the co-morbodity of obesity is commonly resulted from sedentary lifestyle. Thus, physical activity should be considered in PN approach.(89) An optimal personalized diet is not about what an individual is currently doing but to what they should be doing.(38) Some studies showed the inter-individual variability of physical activity benefit on CVD and T2DM risk factors. Some individual even experiences the converse effects on plasma HDL cholesterol, blood pressure, fasting plasma insulin and plasma triglyceride (TG) levels.(90) Thus, physical activity profile should be considered individually to design the diet prescriptions.

Other issue regarding PN is Celiac Disease (CD), a situation of permanent intolerance to gluten/gliadin (prolamin), which results in common heritable chronic inflammatory condition of the small intestine. $C D$ was known as a complex interplay between genetic and environmental factors. $(91,92)$ Prolamin could be found commonly in cereal grains (gliadin in wheat and similar alcohol-soluble proteins in other cereals, secalin in rye, hordein in barley). They act as the environmental stimuli and develop the intestinal damage associated with CD. $(92,93)$ Gliadin contains high proline (Pro or $\mathrm{P}$ ) and is resistant to complete proteolytic digestion by peptidases in the human intestine, make them difficult to be transported across the epithelium. (94) Therefore, they create proline and glutamine (Gln or Q) as toxic oligopeptides accumulation in the small intestine. This lead to toxic effects in genetically susceptible subjects. (95) On the contrary, many dietary components including plant polyphenols, carotenoids and fatty acids, have the 
ability to modulate predisposition to intestinal chronic inflammatory conditions, and will give benefit in nutritional therapy of CD. $(96,97)$ A various mechanisms were involved in their action such as decreasing inflammatory mediator production by utilizing cell signaling and gene expression, reducing the yield of damaging oxidants and improving gut barrier function which lead to anti-inflammatory responses.(98) Hence, by PN, subjects with CD should avoid dietary exposure contain grains but otherwise eat more plant polyphenols, carotenoids and fatty acids, so the inflammatory responses could be avoided.

\section{Conclusion}

Foods are actually a complex mixtures of molecules act in many biochemical pathways simultaneously. They can develop various diseases, or otherwise mediate to solve some conditions. Individual diet recommendation is not simple. Many factors and tools should be involved adequately. The application of integrated omics approaches, together with increasingly detailed nutritional information, lifestyle, physical activity, and microbiome will influence the diet prescription. The advancement application of PN also need a large, appropriate and consistent changes in eating (and other lifestyle) behaviors of individual. However, Application nutrigenomics and nutrigenetics result in a precision nutrition for a more relevant precise personal dietary change recommendation, thus will increase the motivation and sustain to whom the intervention is being delivered.

\section{References}

1. Hyman M. Ultrametabolism: awaken the fat-burning DNA hidden in your body : the simple plan for automatic weight loss. New York; London: Atria Books; 2008.

2. DeBusk RM, Fogarty CP, Ordovas JM, Kornman KS. Nutritional genomics in practice: where do we begin? J Am Diet Assoc. 2005; 105: 589-98.

3. Kaput J, Rodriguez RL. Nutritional genomics: the next frontier in the postgenomic era. Physiol Genomics. 2004; 16: 166-77.

4. Mensink RP, Plat J. Post-genomic opportunities for understanding nutrition: the nutritionist's perspective. Proc Nutr Soc. 2002; 61: 401-4.

5. van Ommen B, Stierum R. Nutrigenomics: exploiting systems biology in the nutrition and health arena. Curr Opin Biotechnol. 2002; 13: 517-21.

6. Ordovas JM, Mooser V. Nutrigenomics and nutrigenetics. Curr Opin Lipidol. 2004; 15: 101-8.

7. van Ommen B. Nutrigenomics: exploiting systems biology in the nutrition and health arenas. Nutr Burbank Los Angel Cty Calif. 2004; 20: 4-8.
8. Müller M, Kersten S. Nutrigenomics: goals and strategies. Nat Rev Genet. 2003; 4: 315-22.

9. Elliott R, Ong TJ. Nutritional genomics. BMJ. 2002; 324: 1438-42.

10. Trayhurn P. Nutritional genomics - "Nutrigenomics." Br J Nutr. 2003; 89: 1-2. doi: 10.1079/BJN2002780.

11. Mathers JC. Nutrigenomics in the modern era. Proc Nutr Soc. 2017; 76: $265-75$.

12. Neeha VS, Kinth P. Nutrigenomics research: a review. J Food Sci Technol. 2013; 50: 415-28.

13. Munshi A, Duvvuri S. Nutrigenomics: Looking to DNA for nutrition advice. Indian J Biotechnol. 2008; 7: 32-40.

14. Kaput J, Swartz D, Paisley E, Mangian H, Daniel WL, Visek WJ. Diet-disease interactions at the molecular level: an experimental paradigm. J Nutr. 1994; 124 (8 Suppl): 1296S-1305S.

15. Park EI, Paisley EA, Mangian HJ, Swartz DA, Wu MX, O’Morchoe $\mathrm{PJ}$, et al. Lipid level and type alter stearoyl CoA desaturase mRNA abundance differently in mice with distinct susceptibilities to dietinfluenced diseases. J Nutr. 1997; 127: 566-73.

16. Black SA. Diabetes, diversity, and disparity: what do we do with the evidence? Am J Public Health. 2002; 92: 543-8.

17. Boden G. Pathogenesis of type 2 diabetes. Insulin resistance. Endocrinol Metab Clin North Am. 2001; 30: 801-15.

18. Nathan DM. Initial management of glycemia in type 2 diabetes mellitus. N Engl J Med. 2002; 347: 1342-9.

19. Wellen KE, Hotamisligil GS. Inflammation, stress, and diabetes. J Clin Invest. 2005; 115: 1111-9.

20. Pavlidis C, Patrinos GP, Katsila T. Nutrigenomics: a controversy. Appl Transl Genomics. 2015;4: 50-3.

21. Nuno N, Heuberger R. Nutrigenetic associations with cardiovascular disease. Rev Cardiovasc Med. 2014; 15: 217-25.

22. Somvanshi PR, Venkatesh KV. A conceptual review on systems biology in health and diseases: from biological networks to modern therapeutics. Syst Synth Biol. 2014; 8: 99-116.

23. Jalili M. Network biology: Describing biological systems by complex networks: Comment on "Network science of biological systems at different scales: A review" by M. Gosak et al. Phys Life Rev. 2018; 24: $159-61$.

24. Abhyankar V, Bland P, Fernandes G. The role of systems biologic approach in cell signaling and drug development responses: a mini review. Med Sci. 2018; 6: 43. doi: 10.3390/medsci6020043.

25. Kitano H. Computational systems biology. Nature. 2002 Nov; 420: 206-10.

26. Kitano H. Systems biology: a brief overview. Science. 2002; 295: 1662-4.

27. Ideker T, Galitski T, Hood L. A new approach to decoding life: systems biology. Annu Rev Genomics Hum Genet. 2001; 2: 343-72.

28. Ideker T, Thorsson V, Ranish JA, Christmas R, Buhler J, Eng JK, et al. Integrated genomic and proteomic analyses of a systematically perturbed metabolic network. Science. 2001; 292: 929-34.

29. Nicholson JK, Wilson ID. Opinion: understanding "global" systems biology: metabonomics and the continuum of metabolism. Nat Rev Drug Discov. 2003; 2: 668-76.

30. Watkins SM, German JB. Toward the implementation of metabolomic assessments of human health and nutrition. Curr Opin Biotechnol. 2002; 13: 512-6.

31. German JB, Roberts MA, Fay L, Watkins SM. Metabolomics and individual metabolic assessment: the next great challenge for nutrition. J Nutr. 2002; 132: 2486-7.

32. German JB, Roberts MA, Watkins SM. Personal metabolomics as a next generation nutritional assessment. J Nutr. 2003; 133: 4260-6.

33. Desiere F. Towards a systems biology understanding of human health: interplay between genotype, environment and nutrition. Biotechnol Annu Rev. 2004; 10: 51-84. 
34. Hesketh J. Personalised nutrition: how far has nutrigenomics progressed? Eur J Clin Nutr. 2013; 67: 430-5.

35. Simopoulos AP. Nutrigenetics/Nutrigenomics. Annu Rev Public Health. 2010; 31: 53-68.

36. Nielsen DE, El-Sohemy A. A randomized trial of genetic information for personalized nutrition. Genes Nutr. 2012; 7: 559-66.

37. Noecker C, Borenstein E. Getting personal about nutrition. Trends Mol Med. 2016; 22: 83-5.

38. Betts JA, Gonzalez JT. Personalised nutrition: What makes you so special? Nutr Bull. 2016; 41: 353-9.

39. Ozdemir V, Suarez-Kurtz G, Stenne R, Somogyi AA, Someya T, Kayaalp SO, et al. Risk assessment and communication tools for genotype associations with multifactorial phenotypes: the concept of "edge effect" and cultivating an ethical bridge between omics innovations and society. Omics J Integr Biol. 2009; 13: 43-61.

40. Patrinos GP, Baker DJ, Al-Mulla F, Vasiliou V, Cooper DN. Genetic tests obtainable through pharmacies: the good, the bad, and the ugly. Hum Genomics. 2013; 7: 17. doi: 10.1186/1479-7364-7-17.

41. Özdemir V, Kolker E. Precision Nutrition 4.0: A big data and ethics foresight analysis--convergence of agrigenomics, nutrigenomics, nutriproteomics, and nutrimetabolomics. Omics J Integr Biol. 2016; 20: 69-75.

42. Trujillo E, Davis C, Milner J. Nutrigenomics, proteomics, metabolomics, and the practice of dietetics. J Am Diet Assoc. 2006; 106: 403-13.

43. Subbiah MTR. Understanding the nutrigenomic definitions and concepts at the food-genome junction. Omics J Integr Biol. 2008; 12: 229-35.

44. Ferguson LR, De Caterina R, Görman U, Allayee H, Kohlmeier M, Prasad C, et al. Guide and position of the International Society of Nutrigenetics/Nutrigenomics on Personalised Nutrition: Part 1 Fields of Precision Nutrition. J Nutr Nutr. 2016; 9: 12-27.

45. Fenech M, El-Sohemy A, Cahill L, Ferguson LR, French TAC, Tai ES, et al. Nutrigenetics and nutrigenomics: viewpoints on the current status and applications in nutrition research and practice. $\mathrm{J}$ Nutr Nutr. 2011; 4: 69-89.

46. Kaput J. Nutrigenomics research for personalized nutrition and medicine. Curr Opin Biotechnol. 2008; 19: 110-20.

47. Jones DP, Park Y, Ziegler TR. Nutritional metabolomics: progress in addressing complexity in diet and health. Annu Rev Nutr. 2012 Aug; 32: 183-202.

48. Fallaize R, Macready AL, Butler LT, Ellis JA, Lovegrove JA. An insight into the public acceptance of nutrigenomic-based personalised nutrition. Nutr Res Rev. 2013; 26: 39-48.

49. Remely M, Lovrecic L, de la Garza AL, Migliore L, Peterlin B, Milagro FI, et al. Therapeutic perspectives of epigenetically active nutrients. Br J Pharmacol. 2015; 172: 2756-68.

50. Martinez JA, Navas-Carretero S, Saris WHM, Astrup A. Personalized weight loss strategies-the role of macronutrient distribution. Nat Rev Endocrinol. 2014; 10: 749-60.

51. Gibney MJ, Walsh MC. The future direction of personalised nutrition: my diet, my phenotype, my genes. Proc Nutr Soc. 2013; 72: 219-25.

52. Martínez A. Perspectives on personalized nutrition for obesity. J Nutr Nutr. 2014; 7: I-III. doi: 10.1159/000365158.

53. Juma S, Imrhan V, Vijayagopal P, Prasad C. Prescribing personalized nutrition for cardiovascular health: are we ready? J Nutr Nutr. 2014; 7: 153-60.

54. Bahcall O. Precision medicine. Nature. 2015; 526: 335. doi: $10.1038 / 526335 \mathrm{a}$.

55. Palou A. From nutrigenomics to personalised nutrition. Genes Nutr. 2007; 2: 5-7

56. Bray MS, Loos RJF, McCaffery JM, Ling C, Franks PW, Weinstock
$\mathrm{GM}$, et al. NIH working group report-using genomic information to guide weight management: From universal to precision treatment. Obes Silver Spring Md. 2016; 24: 14-22.

57. Nielsen DE, Shih S, El-Sohemy A. Perceptions of genetic testing for personalized nutrition: a randomized trial of DNA-based dietary advice. J Nutr Nutr. 2014; 7: 94-104.

58. Nielsen DE, El-Sohemy A. Disclosure of genetic information and change in dietary intake: a randomized controlled trial. PloS One. 2014; 9: e112665. doi: 10.1371/journal.pone.0112665.

59. Arkadianos I, Valdes AM, Marinos E, Florou A, Gill RD, Grimaldi KA. Improved weight management using genetic information to personalize a calorie controlled diet. Nutr J. 2007; 6: 29. doi: 10.1186/1475-2891-6-29.

60. Kohlmeier M, Caterina RD, Ferguson LR, Görman U, Allayee H, Prasad C, et al. Guide and Position of the International Society of Nutrigenetics/Nutrigenomics on Personalized Nutrition: Part 2 Ethics, Challenges and Endeavors of Precision Nutrition. Lifestyle Genomics. 2016; 9: 28-46.

61. Kussmann M, Fay LB. Nutrigenomics and personalized nutrition: science and concept. Pers Med. 2008; 5: 447-55.

62. Fay LB, German JB. Personalizing foods: is genotype necessary? Curr Opin Biotechnol. 2008; 19: 121-8.

63. Gillies PJ. Nutrigenomics: the Rubicon of molecular nutrition. J Am Diet Assoc. 2003; 103 (12 Suppl 2): S50-55.

64. Kohlmeier M, Kohlmeier GZ. Nutrigenetics applying the science of personal nutrition. Amsterdam: Elsevier/Academic Press; 2013.

65. Rao AD, Sun B, Saxena A, Hopkins PN, Jeunemaitre X, Brown NJ, et al. Polymorphisms in the serum- and glucocorticoid-inducible kinase 1 gene are associated with blood pressure and renin response to dietary salt intake. J Hum Hypertens. 2013; 27: 176-80.

66. Ganesh V, Hettiarachchy NS. Nutriproteomics: a promising tool to link diet and diseases in nutritional research. Biochim Biophys Acta. 2012; 1824: 1107-17.

67. Kussmann M, Krause L, Siffert W. Nutrigenomics: where are we with genetic and epigenetic markers for disposition and susceptibility? Nutr Rev. 2010; 68 (Suppl 1): S38-47.

68. Panagiotou G, Nielsen J. Nutritional systems biology: definitions and approaches. Annu Rev Nutr. 2009; 29: 329-39.

69. Grimaldi KA, Look MP, Scioli GA, Clavero JC, Marinos S, Tagaris T. Personal genetics: regulatory framework in Europe from a service provider's perspective. Eur J Hum Genet EJHG. 2011; 19: 382-8.

70. Hogart S. Regulation of genetic tests: an international comparison. In: Castle D, Ries NM, editors. Nutrition and Genomics: Issues of Ethics, Law, Regulation and Communication. 1st ed. Amsterdam: Elsevier; 2016. p.63-83.

71. de Toro-Martín J, Arsenault BJ, Després JP, Vohl MC. Precision nutrition: a review of personalized nutritional approaches for the prevention and management of metabolic syndrome. Nutrients. 2017; 9: 913. doi: 10.3390/nu9080913.

72. Afman L, Müller M. Nutrigenomics: from molecular nutrition to prevention of disease. J Am Diet Assoc. 2006; 106: 569-76.

73. Davis CD, Milner J. Frontiers in nutrigenomics, proteomics, metabolomics and cancer prevention. Mutat Res. 2004; 551: 51-64.

74. Dunn WB, Bailey NJC, Johnson HE. Measuring the metabolome: current analytical technologies. The Analyst. 2005; 130: 606-25.

75. German JB, Roberts MA, Watkins SM. Genomics and metabolomics as markers for the interaction of diet and health: lessons from lipids. J Nutr. 2003; 133 (6 Suppl 1): 2078S-83S.

76. German JB, Bauman DE, Burrin DG, Failla ML, Freake HC, King $\mathrm{JC}$, et al. Metabolomics in the opening decade of the 21 st century: building the roads to individualized health. J Nutr. 2004; 134: 2729-32. 
77. Ordovas JM, Corella D. Nutritional genomics. Annu Rev Genomics Hum Genet. 2004; 5: 71-118.

78. Goni L, Cuervo M, Milagro FI, Martínez JA. A genetic risk tool for obesity predisposition assessment and personalized nutrition implementation based on macronutrient intake. Genes Nutr. 2015; 10: 445. doi: 10.1007/s12263-014-0445-z.

79. Olsen NJ, Ängquist L, Larsen SC, Linneberg A, Skaaby T, Husemoen LLN, et al. Interactions between genetic variants associated with adiposity traits and soft drinks in relation to longitudinal changes in body weight and waist circumference. Am J Clin Nutr. 2016; 104: 816-26.

80. Qi Q, Chu AY, Kang JH, Jensen MK, Curhan GC, Pasquale LR, et al. Sugar-sweetened beverages and genetic risk of obesity. N Engl J Med. 2012; 367: 1387-96.

81. Brunkwall L, Chen Y, Hindy G, Rukh G, Ericson U, Barroso I, et al. Sugar-sweetened beverage consumption and genetic predisposition to obesity in 2 Swedish cohorts. Am J Clin Nutr. 2016; 104: 809-15.

82. Qi Q, Chu AY, Kang JH, Huang J, Rose LM, Jensen MK, et al. Fried food consumption, genetic risk, and body mass index: gene-diet interaction analysis in three US cohort studies. BMJ. 2014; 348: g1610. doi: 10.1136/bmj.g1610.

83. Casas-Agustench P, Arnett DK, Smith CE, Lai CQ, Parnell LD, Borecki IB, et al. Saturated fat intake modulates the association between a genetic risk score of obesity and BMI in two US populations. J Acad Nutr Diet. 2014; 114: 1954-66.

84. Allison DB, Bassaganya-Riera J, Burlingame B, Brown AW, le Coutre J, Dickson SL, et al. Goals in nutrition science 2015-2020. Front Nutr. 2015; 2: 1-13. doi: 10.3389/fnut.2015.00026.

85. Corella D, Coltell O, Mattingley G, Sorlí JV, Ordovas JM. Utilizing nutritional genomics to tailor diets for the prevention of cardiovascular disease: a guide for upcoming studies and implementations. Expert Rev Mol Diagn. 2017; 17: 495-513.
86. Srinivasan B, Lee S, Erickson D, Mehta S. Precision nutrition - review of methods for point-of-care assessment of nutritional status. Curr Opin Biotechnol. 2017; 44: 103-8.

87. Asher G, Sassone-Corsi P. Time for food: the intimate interplay between nutrition, metabolism, and the circadian clock. Cell. 2015; 161: 84-92.

88. Oike H, Oishi K, Kobori M. Nutrients, clock genes, and chrononutrition. Curr Nutr Rep. 2014; 3: 204-12.

89. Hill James O, Wyatt Holly R, Peters John C. Energy balance and obesity. Circulation. 2012; 126: 126-32.

90. Bouchard C, Blair SN, Church TS, Earnest CP, Hagberg JM, Häkkinen $\mathrm{K}$, et al. Adverse metabolic response to regular exercise: is it a rare or common occurrence? PloS One. 2012; 7: e37887. doi: 10.1371/ journal.pone.0037887.

91. Sollid LM, Jabri B. Is celiac disease an autoimmune disorder? Curr Opin Immunol. 2005; 17: 595-600.

92. van Heel DA, West J. Recent advances in coeliac disease. Gut. 2006; 55: 1037-46.

93. Wieser $\mathrm{H}$, Koehler P. The biochemical basis of celiac disease. Cereal Chem. 2008; 85: 1-13. doi: 10.1094/CCHEM-85-1-0001.

94. Jabri B, Kasarda DD, Green PHR. Innate and adaptive immunity: the yin and yang of celiac disease. Immunol Rev. 2005; 206: 219-31.

95. Trynka G, Wijmenga C, van Heel DA. A genetic perspective on coeliac disease. Trends Mol Med. 2010; 16: 537-50.

96. Calder PC, Albers R, Antoine JM, Blum S, Bourdet-Sicard R, Ferns $\mathrm{GA}$, et al. Inflammatory disease processes and interactions with nutrition. Br J Nutr. 2009; 101 (Suppl 1): S1-45.

97. Calder PC. Fatty acids and inflammation: the cutting edge between food and pharma. Eur J Pharmacol. 2011; 668 (Suppl 1): S50-8.

98. Ferretti G, Bacchetti T, Masciangelo S, Saturni L. Celiac disease, inflammation and oxidative damage: a nutrigenetic approach. Nutrients. 2012; 4: 243-57. 